\title{
Good record keeping
}

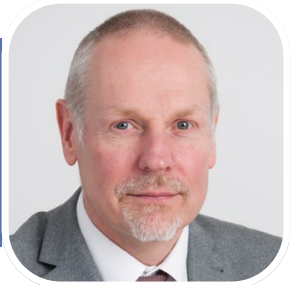

\author{
Leo Briggs, deputy head of the Dental Defence Union (DDU), \\ discusses some of the key issues to consider when managing \\ patient records.
}

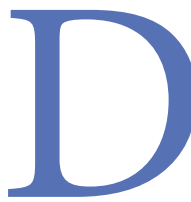

ental records are intended to provide a complete and accurate account of the patient's health and ongoing treatment, to support

continuity of care and minimise the risk of an adverse incident.

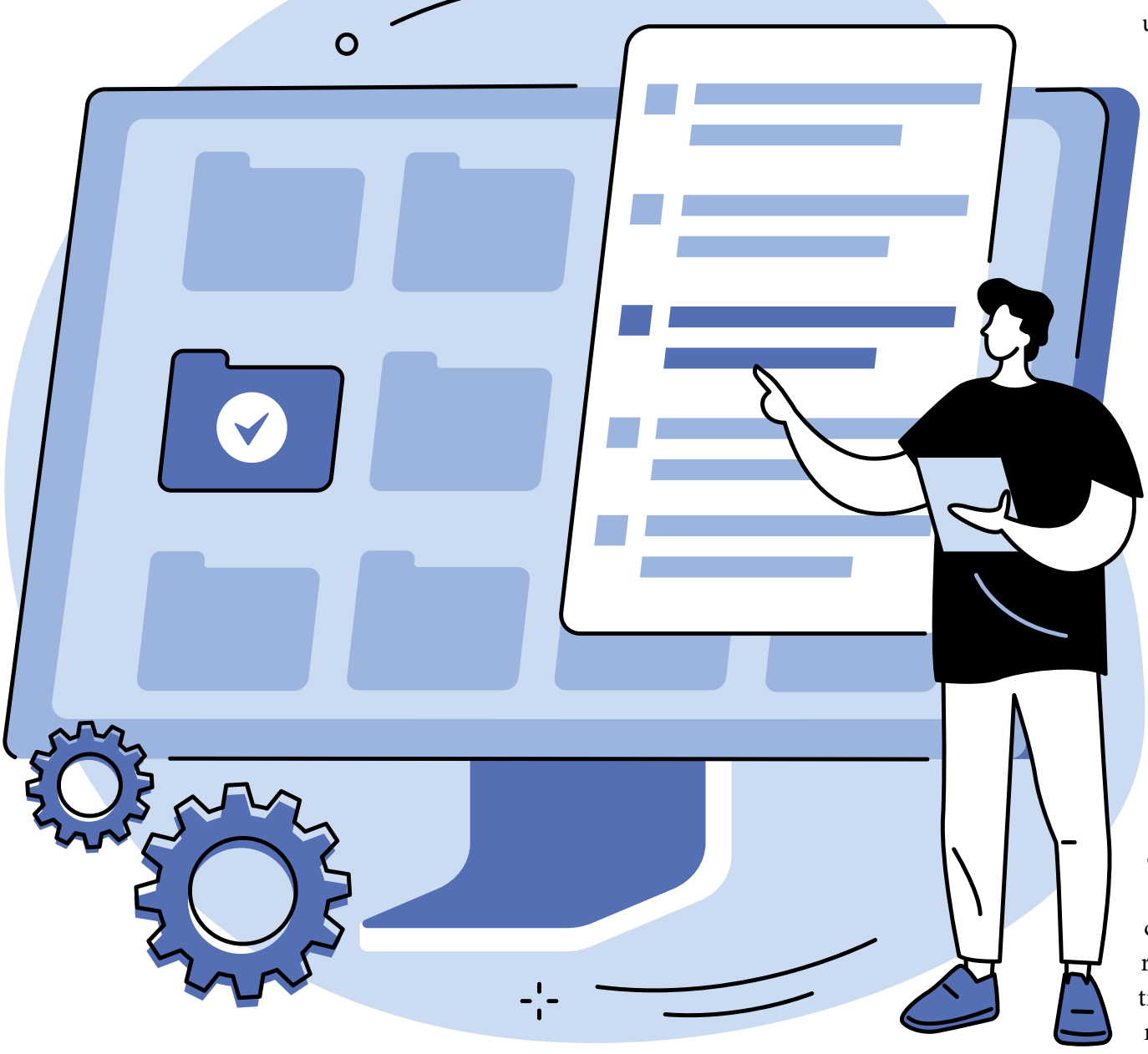

Records are an essential resource for the treating dental professional and for others who might be involved in the care of the patient. Furthermore, good records can provide evidence when defending a dental professional against allegations of clinical negligence. With this in mind, good records should be:

- Contemporaneous: make a record as soon as possible after a patient interaction

- Clear: record your findings carefully and legibly so that they can be understood by anyone who may need to read and interpret them. For example, avoid abbreviations and use one system of dental charting. ${ }^{1}$ It should be clear who made an entry and when

- Complete: record as much detail as possible of all relevant aspects of a patient's appointment. As well as consultations, you should record telephone and email interactions with patients and any information relevant to their care. The exception is complaints correspondence, which should be kept separately because it is not directly relevant to the patient's clinical care

- Concise: records should be just long enough to convey the essential information. Avoid personal comments that could backfire if someone else needs to access the record.

Maintaining electronic records When completing electronic records, it is important that the author is clearly identified and that the date is recorded on the system.

Also, ensure your computer software can produce a hard copy of records and radiographs and can produce a full audit trail of record creation and modification if necessary.

Make sure you have regular backups in 
place to protect against corruption, damage, or destruction of files. However, avoid storing patient identifiable data on personal mobile devices. A back-up of your electronic files should also be held securely off-site in case of accidental loss.

Use secure methods when sending confidential information.
A request does not have to be in writing, but it should be documented, and you should respond promptly.

There may be occasions where a patient asks for a correction to their record. Although the current legislation gives data subjects the right to correct information if it is factually inaccurate or incomplete (the right of rectification), the Information

\section{'Records should be just long enough}

\section{to convey the essential information.}

\section{Avoid personal comments that could}

\section{backfire if someone else needs}

\section{to access the record.'}

\section{Retaining patient records}

Current data protection law states that personal data should be retained for no longer than is necessary but does not set limits.

As a minimum, all patient records should be retained for ten years after the last entry. When records are no longer needed, they must be disposed of in a way that protects patient confidentiality.

\section{Patients' rights}

Patients have the legal right to view their records and obtain copies, known as a subject access request (SAR). Your practice's privacy notice should inform patients of how they can make a SAR, as well as setting out how their information will be processed and securely stored, your legal basis for doing so, and your policy on retention periods.

You are not allowed to charge patients for copies of their records, other than in exceptional cases where a request is 'manifestly unfounded, excessive or repetitive'.
Commissioner's Office (ICO $)^{2}$ has clarified that this does not extend to clinical opinions. There is no requirement to amend an entry because the patient dislikes what has been recorded. Any disagreement over factual matters can be noted, signed and dated in the record.

\section{Disclosing records}

Most patients understand that information will be shared within the direct care team. However, the General Dental Council (GDC) still expects you to explain to patients the circumstances in which you may need to share information with others involved in their healthcare.

It is important to seek the patient's consent and for their decision to be recorded. A patient's wishes should be respected if they decline to give their consent - an exception to this is if there is an overriding public interest (for example, with safeguarding concerns).

Some organisations have the statutory authority to request access to clinical records, although in some cases you might need to inform the patient and ask for their consent. These include:

- Primary care organisations or the NHS Business Services Authority (in England), Dental Practice Board (in Scotland), Central Services Agency (in Northern Ireland)

- Healthcare regulators, such as the CQC, the Parliamentary and Health Service Ombudsman (PHSO)

- The police: disclosure is required under S172 of the Road Traffic Act 1988 and S38B of the Terrorism Act 2000, but otherwise officers might need a valid court order

- The coroner or procurator fiscal in Scotland

- Tax inspectors may request any information or documents it is reasonable for them to have to help them in checking a taxpayer's position under the Finance Act 2008.

If you decide to share a patient's record it is important to document your reasoning as well as whether you have obtained the patient's consent and if required, your justification for disclosing information in the absence of consent. Remember to contact your dental defence organisation if you are unsure about whether to disclose records to a third party.

\section{References}

1. Ashley M. Avoiding errors in dental notation. DDU Journal winter 2017. Available at: https://ddujournal.theddu. com/issue-archive/winter-2017/avoidingerrors-in-dental-notation (accessed August 2021).

2. ICO. Guide to the UK General Data Protection Regulation (UK GDPR). Available at: https://ico.org.uk/fororganisations/guide-to-data-protection/ guide-to-the-general-data-protectionregulation-gdpr/ (accessed August 2021).

https://doi.org/10.1038/s41407-021-0707-8 